\title{
Hour-Specific Total Serum Bilirubin Percentiles for Infants Born at 29-35 Weeks' Gestation
}

\author{
Thivia Jegathesan $^{a, b}$ Joel G. Ray ${ }^{a, c, d}$ Vinod K. Bhutani ${ }^{\mathrm{e}}$ \\ Charles Donald George Keown-Stoneman ${ }^{\mathrm{f}, \mathrm{g}}$ Douglas M. Campbell $\mathrm{a}, \mathrm{b}, \mathrm{h}$ \\ Vibhuti Shah $^{d, h, i}$ Howard Berger ${ }^{c, d}$ Robin Z. Hayeems ${ }^{d, j}$ Michael Sgro a, b, h \\ for the NeoHBC \\ anstitute of Medical Sciences, Temerty Faculty of Medicine, University of Toronto, Toronto, ON, Canada; \\ bDepartment of Pediatrics and Li Ka Shing Knowledge Institute, Unity Health Toronto, St. Michael's Hospital, \\ Toronto, ON, Canada; 'Department of Obstetrics and Gynecology and Li Ka Shing Knowledge Institute, Unity Health \\ Toronto, St. Michael's Hospital, Toronto, ON, Canada; dInstitute of Health Policy, Management and Evaluation, \\ University of Toronto, Toronto, ON, Canada; 'Department of Pediatrics (Neonatology), Stanford School of Medicine, \\ Stanford University, Stanford, CA, USA; ${ }^{A}$ Applied Health Research Centre, Unity Health Toronto, St. Michael's \\ Hospital, Toronto, ON, Canada; 9Dalla Lana School of Public Health, Biostatistics Division, University of Toronto, \\ Toronto, ON, Canada; ' Division of Neonatology, Department of Pediatrics, University of Toronto, Toronto, ON, \\ Canada; 'Department of Paediatrics, Sinai Health, Toronto, ON, Canada; 'ंChild Health Evaluative Sciences, Hospital \\ for Sick Children, Toronto, ON, Canada
}

\section{Keywords}

Hyperbilirubinemia · Quantile regression · Phototherapy · Bilirubin

\begin{abstract}
Introduction: As preterm infants are susceptible to hyperbilirubinemia, they require frequent close monitoring. Prior to initiation of phototherapy, hour-specific total serum bilirubin (TSB) percentile cut-points are lacking in these infants, which led to the current study. Methods: A multi-site retrospective cohort study of preterm infants born between January 2013 and June 2017 was completed at 3 NICUs in Ontario, Canada. A total of 2,549 infants born at 290/7-356/7 weeks' gestation contributed 6,143 pre-treatment TSB levels. Hour-specific TSB percentiles were generated using
\end{abstract}

karger@karger.com www.karger.com/neo

Karger"

GOPEN ACCESS
(C) 2021 The Author(s)

Published by S. Karger AG, Basel

This is an Open Access article licensed under the Creative Commons Attribution-NonCommercial-4.0 International License (CC BY-NC) (http://www.karger.com/Services/OpenAccessLicense), applicable to the online version of the article only. Usage and distribution for commercial purposes requires written permission. quantile regression, further described by degree of prematurity, and among those who subsequently received phototherapy. Results: Among all infants, at birth, hour-specific pre-treatment, TSB percentiles were $36.1 \mu \mathrm{mol} / \mathrm{L}$ (95\% confidence interval $[\mathrm{Cl}]: 34.3-39.3)$ at the $40 \mathrm{th}, 52.3 \mu \mathrm{mol} / \mathrm{L}$ (49.4-55.1) at the 75th, and $79.5 \mu \mathrm{mol} / \mathrm{L}(72.1-89.6)$ at the 95th percentiles. The corresponding percentiles were 39.3 $\mu \mathrm{mol} / \mathrm{L}$ (35.9-43.2), 55.4 $\mu \mathrm{mol} / \mathrm{L}$ (52.1-60.2), and $87.1 \mu \mathrm{mol} / \mathrm{L}$ (Cl 70.5-102.4) prior to initiating phototherapy and 24.4 $\mu \mathrm{mol} / \mathrm{L}$ (20.4-28.8), 35.3 $\mu \mathrm{mol} / \mathrm{L}$ (31.1-41.5), and $52.0 \mu \mathrm{mol} / \mathrm{L}$ (46.1-62.4) among those who did not receive phototherapy. Among infants born at 29-32 weeks, pre-treatment TSB percentiles were $53.9 \mu \mathrm{mol} / \mathrm{L}$ (49.4-61.0) and $95.5 \mu \mathrm{mol} / \mathrm{L}$ (77.5$105.0)$ at the 75 th and 95 th percentiles, with respective values of $48.7 \mu \mathrm{mol} / \mathrm{L}$ (43.0-52.3), and 74.1 $\mathrm{mol} / \mathrm{L}$ (64.8-83.2) for those born at 33-35 weeks' gestation. Conclusion: Hour- 
specific TSB percentiles, derived from a novel nomogram, may inform how bilirubin is described in preterm newborns. Further research of pre-treatment TSB levels is required before clinical consideration.

(c) 2021 The Author(s)

Published by S. Karger AG, Basel

\section{Introduction}

Preterm infants are particularly vulnerable to acute and chronic bilirubin encephalopathy [1]. There have been improvements to the management of hyperbilirubinemia in preterm infants with the introduction of consensus-based guidelines [2]. However, despite this, acute bilirubin encephalopathy and chronic bilirubin encephalopathy continue to occur in preterm infants [3]. In addition, among infants born preterm at $<36$ weeks' gestation, there is a paucity of evidence on safe hour-specific thresholds for total serum bilirubin (TSB) concentrations, especially among those who have not yet received phototherapy $[3,4]$. This issue is compounded by the fact that clinical management largely relies on consensus-based guidelines [5], leaving care providers to use adaptions of these guidelines and clinical discretion in their approach to preterm newborns $[3,5]$.

Contemporary knowledge of the natural history of pre-treatment TSB levels among preterm infants, and the provision of hour-specific statistical cut-points to define hyperbilirubinemia, might influence decision-making about the need for ongoing TSB testing and/or the initiation of phototherapy in this susceptible population. Accordingly, the current study was undertaken to generate hour-specific pre-treatment TSB percentile curves among preterm infants born at $29^{0 / 7}-35^{6 / 7}$ weeks' gestation, including by degree of prematurity, subsequent receipt of phototherapy, and by influential factors such as enteral feeding and laboratory-confirmed ABO incompatibility.

\section{Methods}

This multi-site retrospective cohort study included preterm infants born or transferred to St. Michael's Hospital (January 2013June 2017) and Sinai Health (January 2015-June 2017), in Toronto, ON, Canada, as well as the Hamilton Health Sciences Centre in Hamilton Ontario (January 2014 - June 2017). All healthcare in Ontario is provided under the universal Ontario Health Insurance Plan.

From each of the 3 hospitals, included were preterm infants born at $29^{0 / 7}-35^{6 / 7}$ weeks' gestation. Newborns were excluded who had Rh disease, transferred to a NICU outside of a participating centre without a TSB level done, or who did not have an accessible TSB level in their electronic medical chart. Infants with $\mathrm{Rh}$ disease were excluded, as these infants are identified and managed prenatally through maternal blood work and prenatal screening. The current study focused on care provided to the infant postnatally. Eligible preterm infants born during the study period were identified using the Canadian Neonatal Network and the provincial Better Outcomes Registry and Network databases [6, 7].

Infants who met the main inclusion criteria were further assessed for completeness of their electronic medical charts to obtain pre-treatment TSB levels - defined as any TSB level prior to initiation of phototherapy (among those who went on to receive such phototherapy), or any TSB level otherwise (among those who did not go on to receive phototherapy). Also abstracted was the postnatal age (in hours), phototherapy status, hours of age at phototherapy initiation (if started), gestational age at birth in weeks, and relevant maternal and infant information. All 3 sites routinely screen preterm infants for hyperbilirubinemia from birth and follow local guidelines, adapted from previously published guidelines $[5,8-11]$. Infants with at least one pre-treatment TSB within the first 3 days of birth were included in the final analysis. Infants were also included in a secondary analysis who had laboratory-confirmed $\mathrm{ABO}$ incompatibility, as well as mode of feeding (enteral feeds vs. total parenteral nutrition [TPN] vs. combined enteral + TPN) within the first 10 days of birth.

\section{Data Analyses}

The primary study analysis set out to generate an hour-specific pre-treatment TSB percentile-based nomogram for male and female infants born at $29^{0 / 7}-35^{5 / 6}$ weeks' gestation. Nomograms were developed overall (infants born $29^{0 / 7}-35^{5 / 6}$ weeks' gestation), by subsequent receipt of phototherapy, and by gestational age groups $\left(29^{0 / 7}-32^{6 / 7}\right.$ and $33^{0 / 7}-35^{6 / 7}$ weeks' gestation). All hour-specific pretreatment TSB levels from preterm infants - prior to initiation of phototherapy, and regardless of whether phototherapy was ever started - were plotted in $6 \mathrm{~h}$ increments, from birth up to 5 days thereafter. As used in previous TSB percentile curves for term and near-term infants [12], the 40th, 75th, and 95th percentiles were generated using quantile regression, including quadratic polynomials for age (in hours). The quantreg package in $\mathrm{R}$ version 4.0.3 was used for fitting all quantile regression models [13]. Modelling accounting for repeated measures within an infant and 1,000 bootstrap resamples of participants were used to generate $95 \%$ confidence intervals (CIs) [14]. Quantile regression was used to generate pre-treatment TSB levels because it allows one to evaluate the relation of independent variables across a full range of continuous dependent variables, rather than a conditional mean [15]. Missing data were labelled as "unknown," and accounted for in relevant sub-analyses.

To assist with the clinical interpretation of the percentile-based nomogram, the mean pre-treatment peak TSB, and the time of an infant's pre-treatment peak TSB from birth to $72 \mathrm{~h}$ of age were calculated for all newborns, by receipt of phototherapy, by gestational age groupings, by laboratory-confirmed $\mathrm{ABO}$ incompatibility, as well as by mode of feeding - as described above. To determine differences in infants' mean pre-treatment peak TSB, and hours of age of pre-treatment peak TSB within the first $72 \mathrm{~h}$ since birth, a one-way ANOVA was conducted for these aforementioned neonatal factors, with statistical significance $p$ values $<0.01$. Finally, to determine differences in the proportion of infants who subsequently received phototherapy, a $\chi^{2}$ analysis was conducted for the above-mentioned neonatal factors, tested at a $p$ value $<0.01$. All statistical analyses were conducted using R version 4.0.3 and SPSS 27 for Mac OS $[13,16]$. 
Table 1. Neonatal and maternal characteristics of 2,549 preterm infants included in the study by prematurity groups

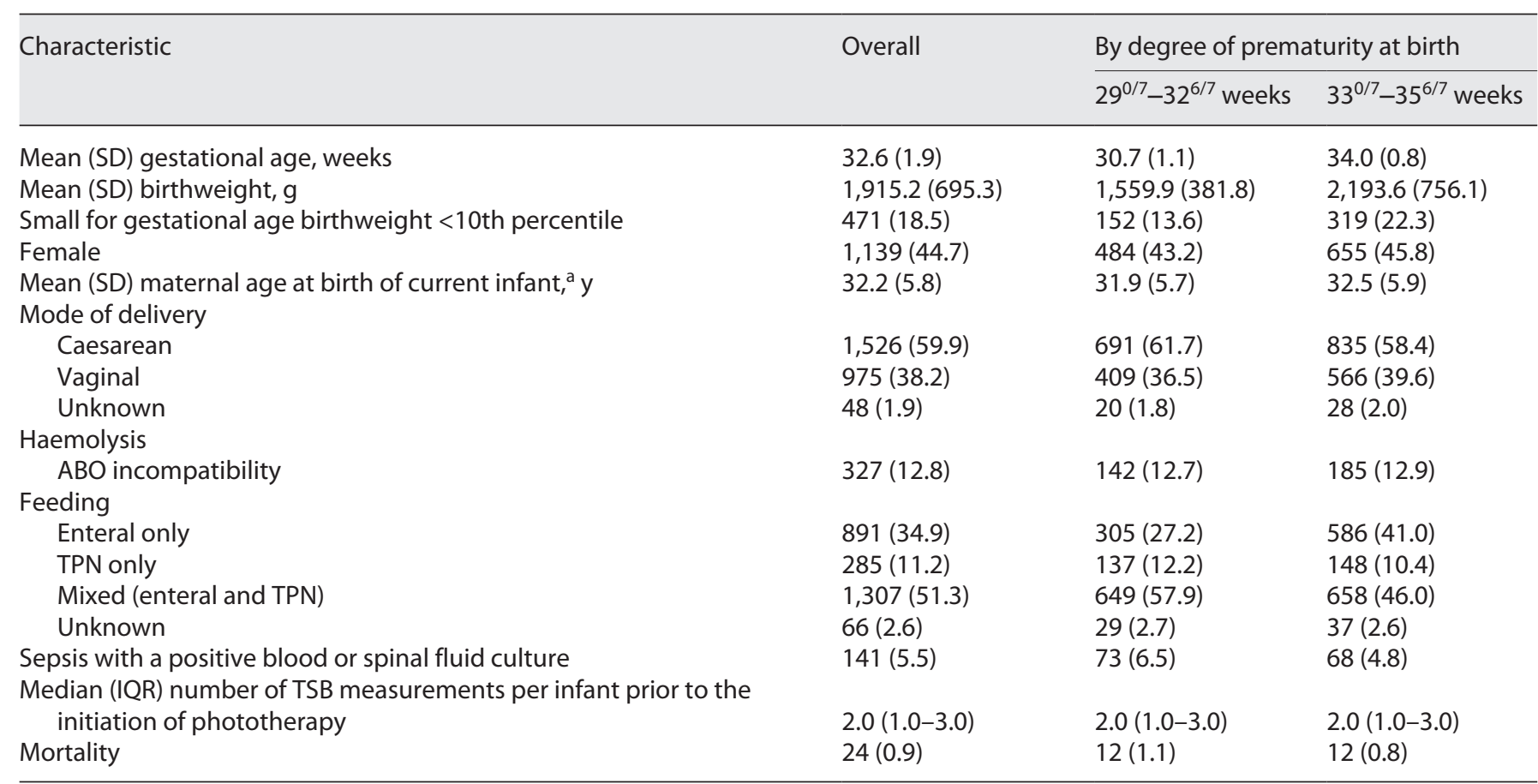

All data are presented as a number (\%) unless otherwise indicated. SD, standard deviation; TPN, total parenteral nutrition. ${ }^{\mathrm{a}} \mathrm{Maternal}$ age missing in $<1 \%$ of cohort overall and by degree of prematurity.

\section{Sample Size Calculation}

The sample size was calculated based on assumptions from previous nomograms for pre-treatment TSB levels in term and nearterm infants [17]. Using the methods for reference limits by Bellera and Hanley [18], in order to obtain $2.5 \%$ and $97.5 \%$ reference limits, with a relative margin of error of $10 \%$ for a Gaussian distribution, a minimum sample size of 448 preterm infants was required. Since the TSB curve-by-time was further stratified by infants born at 29-32 and 33-35 weeks' gestation, and while accounting for a potentially $20 \%$ loss-to-follow-up, a minimum of 537 infants was deemed necessary for the 29-32 and 33-35 weeks' gestation age groups, respectively.

\section{Results}

Out of 2,954 preterm infants born at $29^{0 / 7}-35^{6 / 7}$ weeks' gestation, 2,549 infants had at least one pre-treatment TSB level available from birth to $72 \mathrm{~h}$ of age (online suppl. Fig. 1; for all online suppl. material, see www.karger.com/ doi/10.1159/000519496). The mean (standard deviation [SD]) gestational age and birthweight of the study population was 32.6 (1.9) weeks and 1,915.2 (695.3) g, respectively (Table 1).

\section{All Infants}

In the creation of the main nomogram, 2,549 infants contributed a total of 6,143 hour-specific pre-treatment TSB measures (Fig. 1a). The pre-treatment estimated TSB percentiles at birth were $36.1 \mu \mathrm{mol} / \mathrm{L}$ (95\% CI: 34.3-39.3) at the $40 \mathrm{th}, 52.3 \mu \mathrm{mol} / \mathrm{L}(95 \% \mathrm{CI}: 49.4-55.1)$ at the 75 th, and $79.5 \mu \mathrm{mol} / \mathrm{L}(95 \% \mathrm{CI}: 72.1-89.6)$ at the 95th percentiles (Table 2). The corresponding nomogram-estimated rate of rise of TSB from birth was similar across all 3 percentiles. The estimated change in the rate of rise of TSB then diminished with advancing age after birth (Table 2).

Overall, the estimated pre-treatment TSB level percentiles peaked at $90.6 \mathrm{~h}$ of age for the 40th percentile and peaked later at the 75 th and 95th percentile (Table 2; Fig. 1a). Upon limiting the data to the first $72 \mathrm{~h}$ of age, the mean (SD) peak TSB was $142.0 \mu \mathrm{mol} / \mathrm{L}(37.9)$ at a mean (SD) of 41.7 (17.3) hours of age (Table 3$).$

\section{By Subsequent Receipt of Phototherapy}

A total of 696 infants $(27.3 \%)$ did not receive phototherapy during their hospitalization, and they provided 2,306 hour-specific TSB levels (Fig. 1b). There were 1,853 infants $(72.7 \%)$ who subsequently received phototherapy at a mean (SD) of 46.5 (26.9) hours of age, and they pro- 


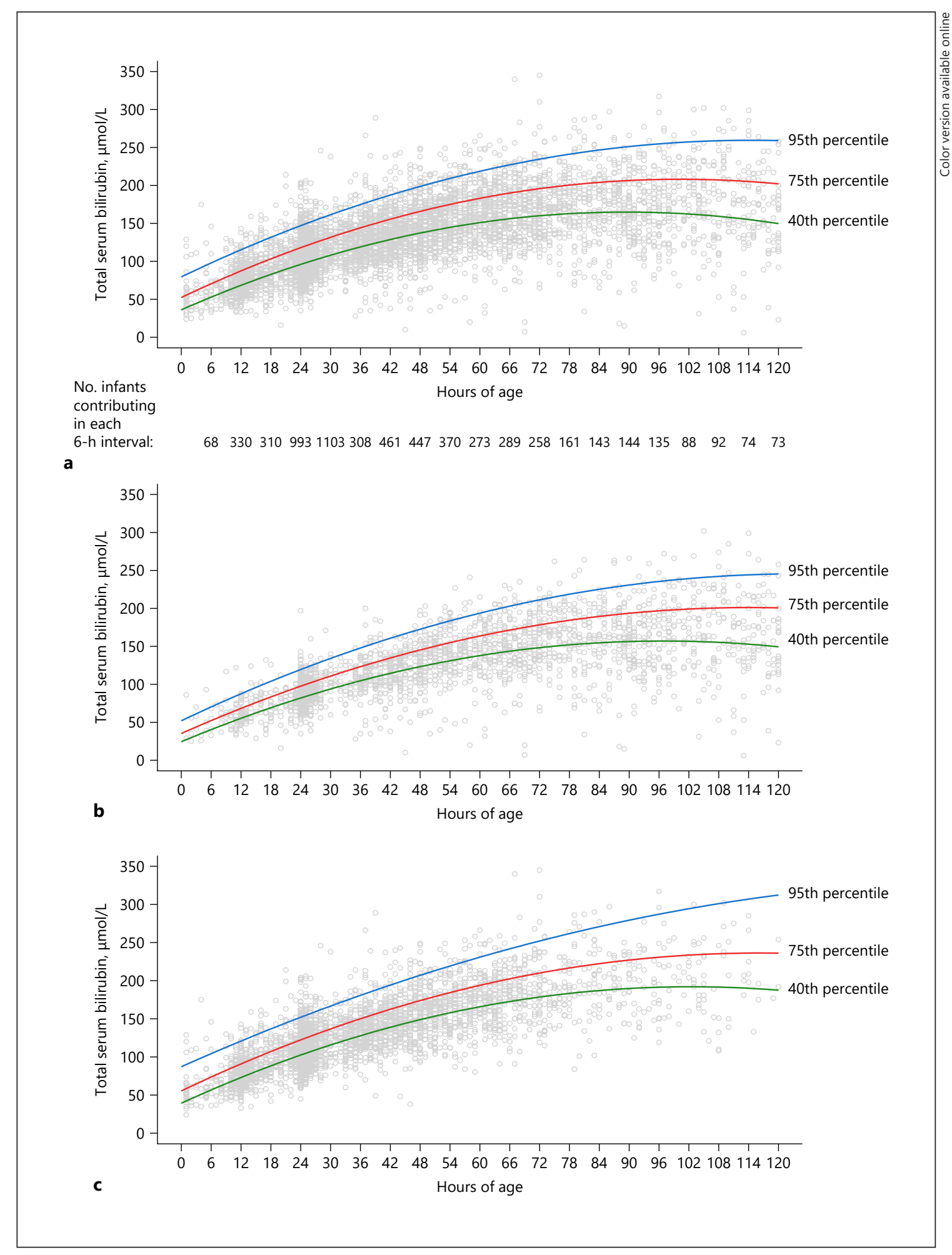

Fig. 1. Hour-specific pre-treatment TSB percentile-based curves among preterm infants born at 29-35 weeks' gestation: (a) overall $(n=2,549)$, (b) among infants not subsequently administered phototherapy $(n=696)$, and (c) prior to the initiation phototherapy $(n=1,853)$. Pre-treatment TSB levels refer to TSB levels prior to phototherapy among those administered phototherapy and any TSB levels among those not administered phototherapy. To convert TSB levels to $\mathrm{mg} / \mathrm{dL}$, divide by 17.1. Frames around graphs were removed. TSB, total serum bilirubin. 

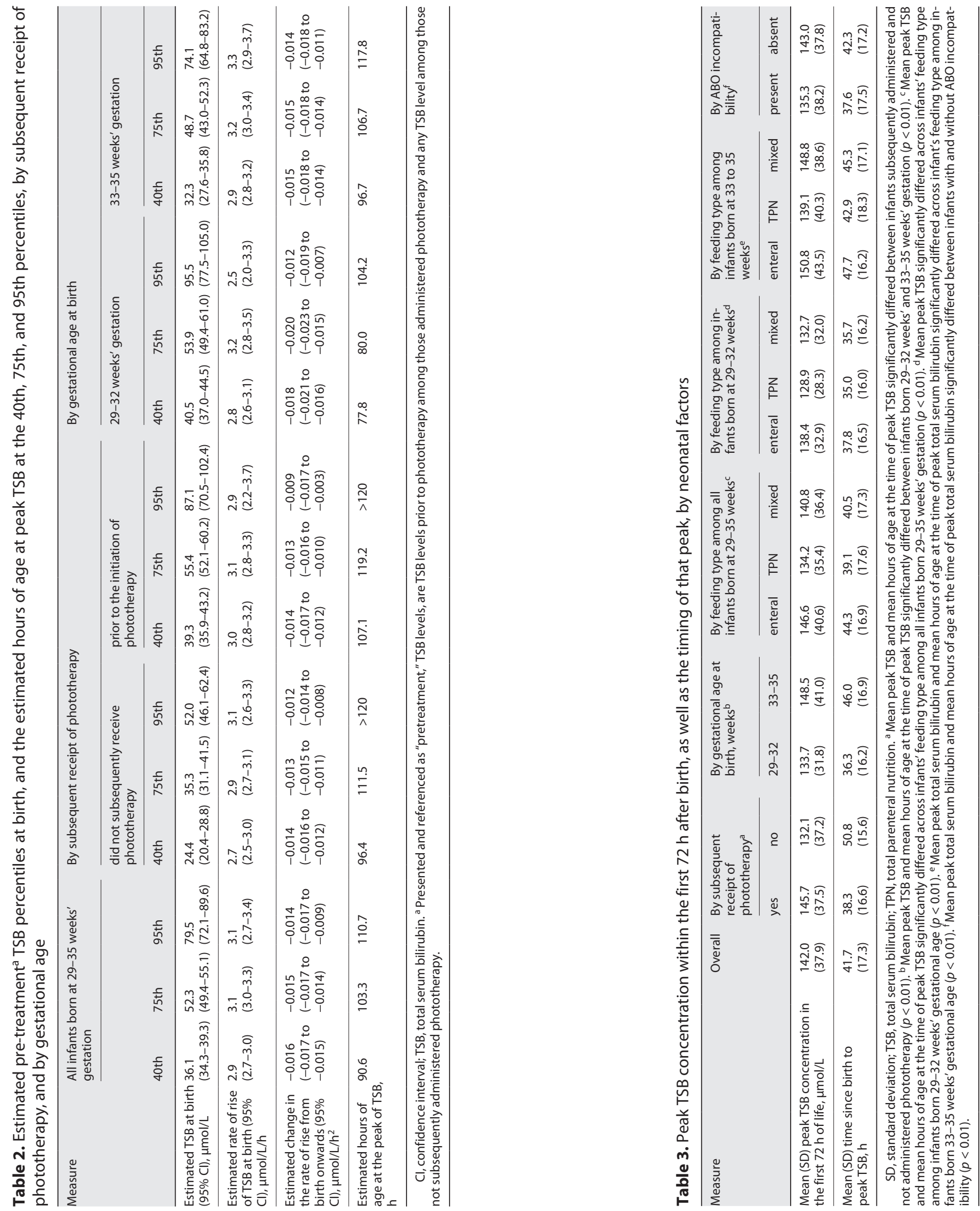


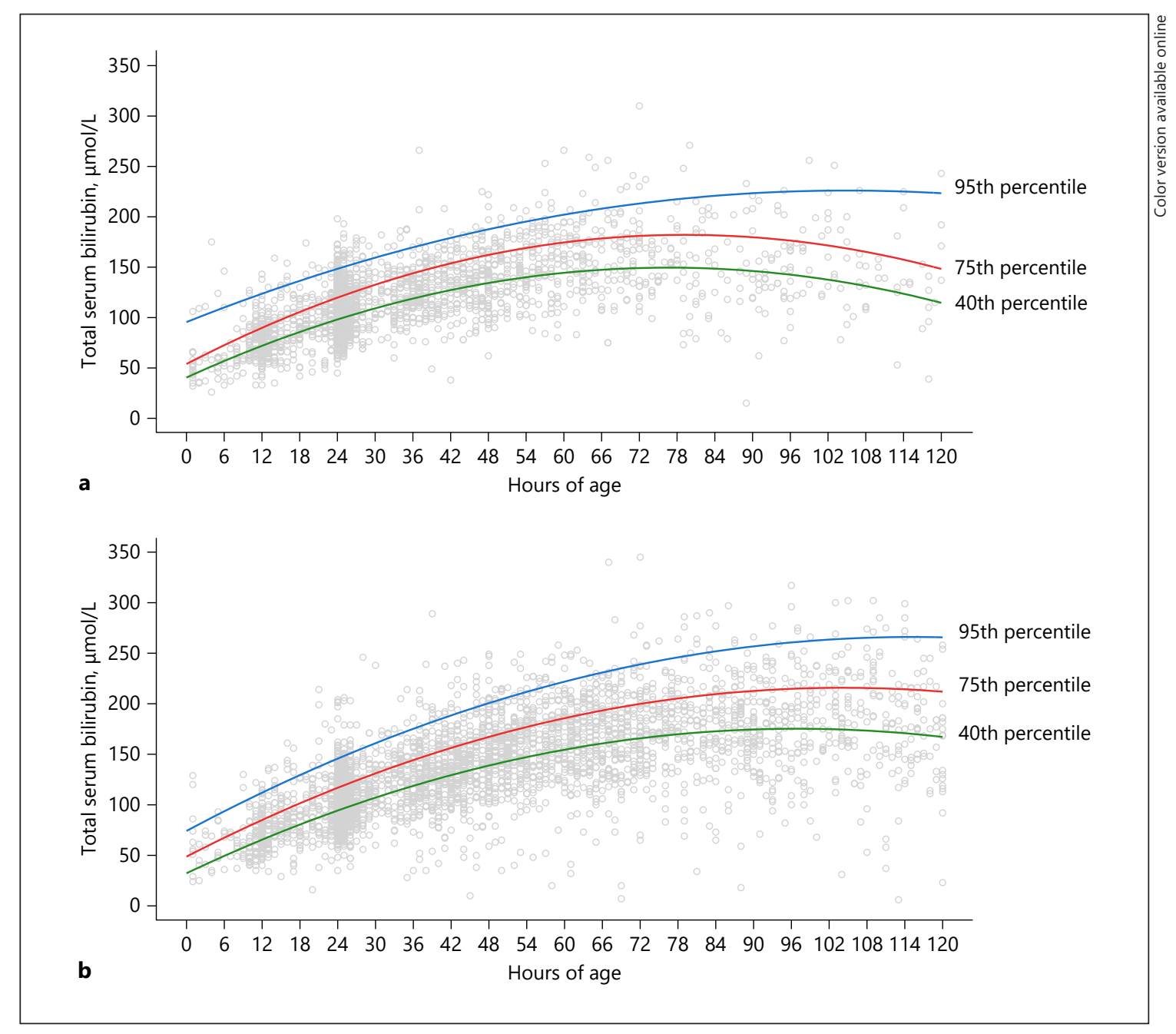

Fig. 2. Hour-specific pre-treatment TSB percentile-based curves among preterm infants born at (a) 29-32 weeks' gestation $(n=1,120)$ and (b) 33-35 weeks' gestation $(n=1,429)$. Pre-treatment TSB levels refer to TSB levels prior to phototherapy among those administered phototherapy and any TSB levels among those not administered phototherapy. To convert TSB levels to $\mathrm{mg} / \mathrm{dL}$, divide by 17.1. Frames around graphs were removed. TSB, total serum bilirubin.

vided 3,837 hour-specific pre-treatment TSB levels (Fig. 1c). Their overall pre-treatment TSB levels at birth at the 40th, 75th, and 95th percentiles were higher than the preterm infants who did not receive phototherapy (Table 2). Within the first $72 \mathrm{~h}$ of age, those administered phototherapy had a higher mean peak in TSB (145.7 $\mu \mathrm{mol} / \mathrm{L}$ vs. $132.1 \mu \mathrm{mol} / \mathrm{L} ; p<0.01)$, which occurred significantly earlier (38.3 vs. 50.8 h; $p<0.01$ ) (Table 3 ).

\section{By Degree of Prematurity}

A total of 1,120 infants were born at $29^{0 / 7}-32^{6 / 7}$ weeks' gestation, providing 2,313 pre-treatment TSB levels (Fig. 2a), and 1,429 infants were born at $33^{0 / 7}-35^{6 / 7}$ weeks, with a total of 3,830 pre-treatment TSB levels (Fig. 2b). For those born at 29-32 weeks' gestation, estimated pretreatment TSB percentiles at birth were $53.9 \mu \mathrm{mol} / \mathrm{L}(95 \%$ CI: 49.4-61.0) at the 75th percentile and $95.5 \mu \mathrm{mol} / \mathrm{L}$ (95\% CI: 77.5-105.0) at the 95th percentile (Table 2). Among those born at 33-35 weeks' gestation, the respective values were $48.7 \mu \mathrm{mol} / \mathrm{L}$ (95\% CI: 43.0-52.3) and 74.1 $\mu \mathrm{mol} / \mathrm{L}$ (95\% CI: 64.8-83.2) (Table 2).

The estimated TSB rate of rise from birth was generally similar between the 2 gestational age groups, except at the 95th percentile, where the estimated TSB rate of rise was higher among infants born at 33-35 weeks' gestation than those born at 29-32 weeks (Table 2). Pre-treatment 


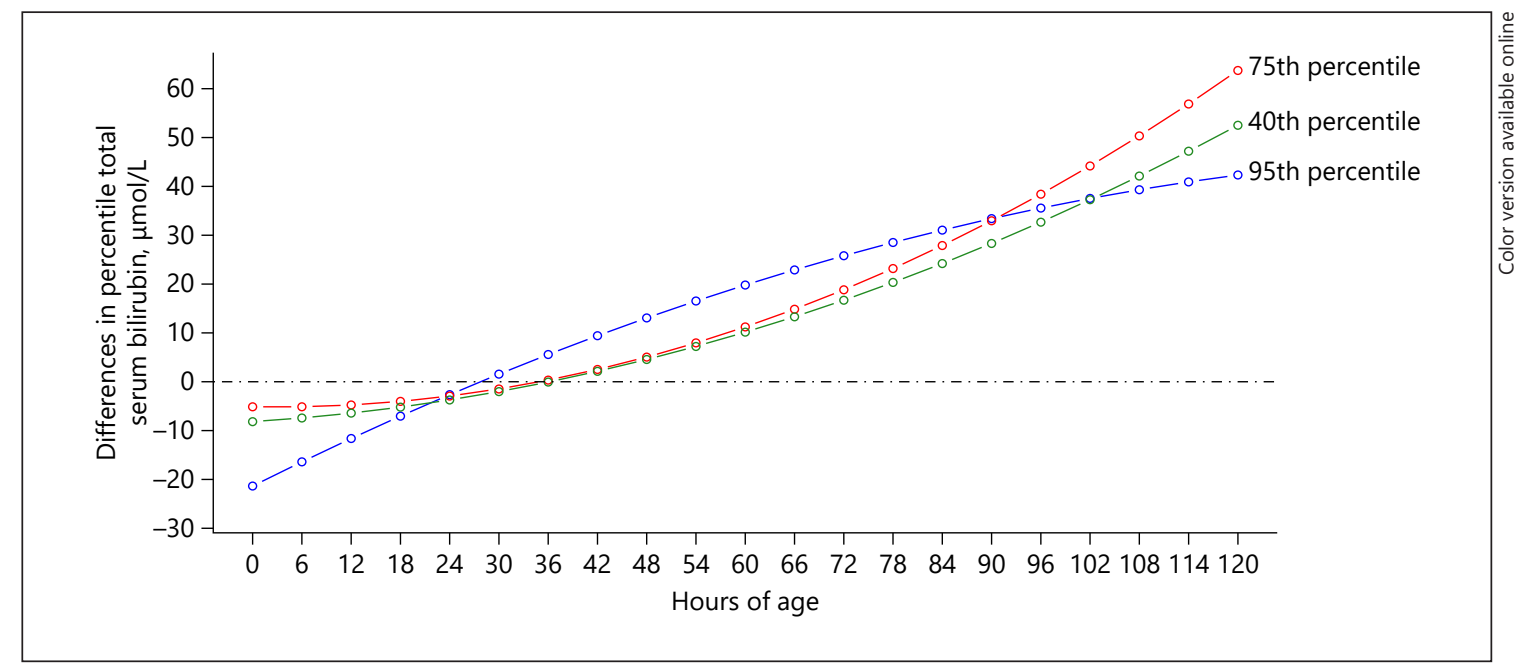

Fig. 3. Pre-treatment TSB percentile net differences between infants born at 33-35 weeks' gestation minus those born at 29-32 weeks' gestation. Pre-treatment TSB levels refer to TSB levels prior to phototherapy among those administered phototherapy and any TSB levels among those not administered phototherapy. To convert mean TSB differences to $\mathrm{mg} / \mathrm{dL}$, divide by 17.1. Frames around graphs were removed. TSB, total serum bilirubin.

TSB percentiles peaked earlier among the latter than the former (Table 2; Fig. 2a, b). Within the first $72 \mathrm{~h}$ after birth, mean pre-treatment TSB level peaked significantly earlier in infants born at 29-32 weeks than those born later (36.3 vs. $46.0 \mathrm{~h} ; p<0.01)$ and with a significantly lower mean peak TSB level among the former versus latter ( 133.7 vs. $148.5 \mu \mathrm{mol} / \mathrm{L} ; p<0.01)$ (Table 3$)$. Furthermore, significantly more infants born at 29-32 weeks' gestation received phototherapy than those born at 33-35 weeks $(91.1 \%$ vs. $58.3 \%$; $p<0.01)$ (online suppl. Table 1 ).

Estimated pre-treatment TSB level percentile differences between infants born at 33-35 weeks' gestation and those born at 29-32 weeks' gestation widened with time since birth, especially after $24 \mathrm{~h}$ of age (Fig. 3). Actual mean differences are shown in online supplementary Fig. 2.

\section{By Feeding Type}

When stratified by feeding type or laboratory-confirmed $\mathrm{ABO}$ incompatibility, there was an insufficient number of pre-treatment TSB levels to generate hourspecific pre-treatment TSB percentile curves. Overall, among all infants, the mean peak TSB between birth and $72 \mathrm{~h}$ was significantly higher in infants exclusively receiving enteral nutrition $(146.6 \mu \mathrm{mol} / \mathrm{L})$ than those receiving TPN $(134.2 \mu \mathrm{mol} / \mathrm{L})$, or a combination of both $(140.8$ $\mu \mathrm{mol} / \mathrm{L})(p<0.01)$ (Table 3$)$. Significantly more infants who were on TPN - with or without enteral feeds - were administered phototherapy than those exclusively receiving enteral feeds (online suppl. Table 1).

\section{Peak Pre-treatment TSB by ABO Incompatibility}

Among all 2,549 infants, 327 (12.8\%) had laboratoryconfirmed ABO incompatibility. Those with ABO incompatibility had their TSB peak at a significantly lower concentration, and earlier, than infants without $\mathrm{ABO}$ incompatibility (Table 3).

\section{Discussion}

This multi-site retrospective cohort study produced a novel hour-specific, pre-treatment TSB percentile-based nomogram for preterm infants born at $29^{0 / 7}-35^{6 / 7}$ weeks' gestation. Hour-specific pre-treatment TSB levels differed between infants born at $33^{0 / 7}-35^{6 / 7}$ and $29^{0 / 7}-32^{6 / 7}$ weeks' gestation. Degree of prematurity and infant nutrition was each significantly associated with the subsequent initiation of phototherapy.

This is one of the first and largest studies to estimate hour-specific percentiles of pre-treatment TSB levels in preterm infants born at $29^{0 / 7}-35^{6 / 7}$ weeks' gestation. One previous study was limited to fewer than 1,000 very low birthweight preterm infants [4]. Other studies relied on perceived risk of hyperbilirubinemia in preterm infants, rather than pre-treatment TSB levels directly from preterm infants $[3,5]$. Finally, this is one of the first studies to describe the influence of feeding type on TSB levels and phototherapy initiation in preterm infants. 
This study has some limitations. Firstly, fewer infants at more extreme prematurity were included, as they are more likely to undergo early initiation of phototherapy. Accordingly, no infants born before 29 weeks' gestation were included. In our study, initiation of phototherapy was at the discretion of the clinical care team, rather than by a defined study protocol. Nevertheless, a large number of pre-treatment TSB levels were collected up to $120 \mathrm{~h}$ after birth. Secondly, information about infant feeding was limited to the first 10 days of life and lacked details about the volume and type of enteral feeds or TPN. Certainly, other approaches to the management of hyperbilirubinemia and feeding practices in preterm infants could have generated somewhat different TSB nomograms and TSB percentiles than those based on Canadian infants from 3 nearby clinical centres. Thirdly, our study was limited to investigating the impact of initiating phototherapy, gestational age at birth, $\mathrm{ABO}$ incompatibility, and feeding on TSB levels as these are common clinical factors investigated in preterm infants. Understandably, the overall health of the infant may also impact TSB levels. However, as this study is the first step in understanding TSB levels, we limited our study to these 3 factors. Further research should be conducted to investigate additional clinical factors.

Currently, TSB levels are used to screen for hyperbilirubinemia and decide on whether or not to initiate phototherapy [5]. Our study compared estimated TSB percentile levels at birth among preterm infants by receipt of phototherapy and gestational age group at birth. Accordingly, we noted higher estimated pre-treatment TSB percentiles at birth among preterm infants who were administered phototherapy than those who did not receive phototherapy. This was consistent with previous research that noted higher TSB levels at $12 \mathrm{~h}$ of age among those who were at higher risk of developing significant hyperbilirubinemia [19]. In addition, our study was limited to TSB levels prior to the initiation of phototherapy.

As preterm infants are known to be at a higher risk for severe hyperbilirubinemia, they tend to receive treatment at lower TSB thresholds than term infants $[3,5,20]$. In our study, within the first $72 \mathrm{~h}$ of age, TSB peaked earlier in infants born more premature - often before $48 \mathrm{~h}-$ which is consistent with previous research on predictors of severe hyperbilirubinemia $[5,20]$. However, this observed early peak in TSB levels among more premature infants was likely due to the fact that many received phototherapy soon thereafter $[21,22]$.

Delayed hyperbilirubinemia has been reported in preterm infants born at 34-35 weeks' gestation [23]. All pre-

Total Serum Bilirubin Percentiles for

Moderate Preterm Infants term infants could experience delayed hyperbilirubinemia. We observed that infants at the 95th percentile of TSB continued to experience a TSB rise up to $110 \mathrm{~h}$ of age, for example. This would suggest that TSB should continue to be monitored beyond $72 \mathrm{~h}$ of age in all preterm infants, especially among infants whose TSB is near the 95th percentile.

As seen elsewhere [21, 22], gestational age had a significant impact on TSB levels and initiation of phototherapy. Pre-treatment TSB percentile curves differed significantly between infants born at 29-32 weeks' or 33-35 weeks' gestation, suggesting that 2 different nomograms might be needed for these age groups $[3,5,20]$.

Although the impact of ABO incompatibility on the risk of hyperbilirubinemia in preterm infants has been well studied, that of infant nutrition has not $[24,25]$. After being processed by the liver, bilirubin is excreted into the gastrointestinal tract, and therefore, gastrointestinal tract abnormalities and motility issues can further affect bilirubin clearance [26]. In our study, more infants who received TPN, either alone, or with enteral feeds, received phototherapy and experienced an earlier peak in TSB than those solely receiving enteral feeds. A potential reason for the increased use of phototherapy among infants on TPN may be illness; however, further research is required to determine whether illness or feeding type alone can be attributed to the difference in TSB levels and receipt of phototherapy seen by feeding type. Hence, early nutrition may be an additional factor to consider when determining the risk of hyperbilirubinemia.

For preterm infants, our study provides clinicians and policy-makers with novel information about hourly trends in pre-treatment TSB levels in preterm infants. Analogous to research determining the optimal threshold for oxygen and carbon dioxide in preterm infants, there is also a need to identify which hourly bilirubin threshold is safest, short-term and long-term [27, 28]. In line with previous research, a clinical trial might compare initiation of phototherapy at the lower versus higher pre-treatment TSB percentiles described herein [29].

\section{Conclusion}

The American Academy of Pediatrics has called for development of guidelines for the management of jaundice in preterm infants. In response, we generated hourspecific TSB percentiles and nomogram plots [5]. While the current nomograms offer a first step in reliably understanding bilirubin levels in preterm infants, further re- 
search is needed to determine their predictive ability and association with bilirubin toxicity. This will assist in identifying infants at higher risk of significant hyperbilirubinemia.

\section{Acknowledgments}

Some members of the Neonatal Hyperbilirubinemia Consortium (NeoHBC) are Jillian Baker, Jeffrey Antwi, Shangari Baleswaran, Matthew Barozzino, Tony Barozzino, Maria Casalino, Sohail Cheema, Mary Debono, Danielle Dobberstein, Maria Ghobrial, Aasha Gnanalingam, Aaditeya Jhaveri, Sharmilaa Kandasamy, Melissa Librach, Dishaben Prajapati, Saisujani Rasiah, Tavpritesth Sethi, Jennifer Twiss, and Gayathri Visvanathaiyer.

\section{Statement of Ethics}

Institutional Research Ethics Board approval was obtained at all 3 participating sites (St. Michael's Hospital REB \#17-225). Informed consent was not required since this was a retrospective chart review.

\section{Conflict of Interest Statement}

The authors have no conflicts of interest to declare.

\section{Funding Sources}

This study was funded by Drager Medical Systems Inc, Germany, and the University of Toronto Open Fellowship Award, Toronto Canada.

\section{Author Contributions}

Thivia Jegathesan, Dr. Joel Ray, and Dr. Michael Sgro conceptualized the design of the study, drafted the initial manuscript, and reviewed and revised the manuscript.

Dr. Vinod Bhutani, Dr. Charles Donald George Keown-Stoneman, Dr. Douglas Campbell, Dr. Howard Berger, and Dr. Robin Hayeems assisted in the design of the study, assisted with the analyses, and critically reviewed and revised the manuscript.

Dr. Vibhuti Shah assisted in the design of the study, coordinated data collection, and reviewed and revised the manuscript.

\section{Data Availability Statement}

All data analysed during this study are included in this article in aggregate form. De-identified individual participant data will not be made available due to privacy restrictions.

\section{References}

1 Crosse VM, Meyer TC, Gerrard JW. Kernicterus and prematurity. Arch Dis Child. 1955; 30(154):501-8

2 Maisels MJ, Watchko JF. Treatment of jaundice in low birthweight infants. Arch Dis Child Fetal Neonatal Ed. 2003;88(6):F45963.

3 Bhutani VK, Wong RJ. Bilirubin neurotoxicity in preterm infants: risk and prevention. J Clin Neonatol. 2013;2(2):61-9.

4 Hahn S, Bührer C, Schmalisch G, Metze B, Berns M. Rate of rise of total serum bilirubin in very low birth weight preterm infants. Pediatr Res. 2020;87:1039-44.

5 Maisels MJ, Watchko JF, Bhutani VK, Stevenson DK. An approach to the management of hyperbilirubinemia in the preterm infant less than 35 weeks of gestation. J Perinatol. 2012; 32(9):660-4.

6 The Canadian Neonatal Network (CNN). The Canadian Neonatal Network Toronto. Canadian Neonatal Network; 2009. Available from: http://www.canadianneonatalnetwork. org/portal/.

7 Better Outcomes Registry \& Network (BORN). BORN Ontario. Ottawa: BORN Ontario; 2019. Available from: https://www.bornontario.ca/en/index.aspx.
8 National Institute for Health and Clinical Excellence. Neonatal Jaundice National Institute for Health and Clinical Excellence; 2010. Available from: https://www.nice.org.uk/guidance/CG98.

9 Morris BH, Oh W, Tyson JE, Stevenson DK, Phelps DL, O'Shea TM, et al. Aggressive vs. conservative phototherapy for infants with extremely low birth weight. N Engl J Med. 2008;359(18):1885-96.

10 Canadian Pediatric Society. Guidelines for detection, management and prevention of hyperbilirubinemia in term and late preterm newborn infants (35 or more weeks' gestation) - summary. Paediatr Child Health. 2007;12(5):401-18.

11 American Academy of Pediatrics Subcommittee on Hyperbilirubinemia. Management of hyperbilirubinemia in the newborn infant 35 or more weeks of gestation. Pediatrics. 2004;114(1):297-316.

12 Bhutani VK, Johnson L, Sivieri EM. Predictive ability of a predischarge hour-specific serum bilirubin for subsequent significant hyperbilirubinemia in healthy term and nearterm newborns. Pediatrics. 1999;103(1):6-14.

13 R Development Core Team R. A language and environment for statistical computing. Vienna, Austria: R Foundation for Statistical Computing; 2009.
14 Karlsson A. Bootstrap methods for bias correction and confidence interval estimation for nonlinear quantile regression of longitudinal data. J Stat Comput Simul. 2009;79(10):1205-18.

15 Marrie RA, Dawson NV, Garland A. Quantile regression and restricted cubic splines are useful for exploring relationships between continuous variables. J Clin Epidemiol. 2009; 62(5):511-7.e1.

16 IBM Corp. IBM Statistics for Machintosh. 27 ed. IBM SPSS; 2020.

$17 \mathrm{Yu} \mathrm{ZB}$, Han SP, Chen C. Bilirubin nomograms for identification of neonatal hyperbilirubinemia in healthy term and late-preterm infants: a systematic review and meta-analysis. World J Pediatr. 2014;10(3):211-8.

18 Bellera CA, Hanley JA. A method is presented to plan the required sample size when estimating regression-based reference limits. J Clin Epidemiol. 2007;60(6):610-5.

19 Mayer I, Gursoy T, Hayran M, Ercin S, Ovali F. Value of twelfth hour bilirubin level in predicting significant hyperbilirubinemia in preterm infants. J Clin Med Res. 2014;6(3):1906.

20 Bhutani VK, Wong RJ, Stevenson DK. Hyperbilirubinemia in preterm neonates. Clin Perinatol. 2016;43(2):215-32. 
21 Mreihil K, Benth JŠ, Stensvold HJ, Nakstad B, Hansen TWR; Norwegian Neonatal Network. Phototherapy is commonly used for neonatal jaundice but greater control is needed to avoid toxicity in the most vulnerable infants. Acta Paediatr. 2018;107(4):611-9.

22 Mukherjee D, Coffey M, Maisels MJ. Frequency and duration of phototherapy in preterm infants $<35$ weeks gestation. J Perinatol. 2018;38(9):1246-51.

23 Whyte R. Safe discharge of the late preterm infant. Paediatr Child Health. 2010;15(10): 655-66.
24 Khurana R, Batra P, Faridi M, Khan N. Revisiting $\mathrm{ABO}$ incompatibility as a risk factor for significant neonatal hyperbilirubinemia. Trop Doct. 2019;49(3):201-4.

25 Yogev-Lifshitz M, Leibovitch L, SchushanEisen I, Taran C, Strauss T, Maayan-Metzger A. Indication of mild hemolytic reaction among preterm infants with $\mathrm{ABO}$ incompatibility. Pediatr Blood Cancer. 2016;63(6): 1050-3.

26 Moerschel SK, Cianciaruso LB, Tracy LR. A practical approach to neonatal jaundice. Am Fam Physician. 2008;77(9):1255-62.
27 Carlo WA, Carlo WA, Finer NN, Walsh MC, Rich W, Gantz MG, et al. Target ranges of oxygen saturation in extremely preterm infants. N Engl J Med. 2010;362(21):1959-69.

28 Khan A, Qurashi M, Kwiatkowski K, Cates D, Rigatto $\mathrm{H}$. Measurement of the $\mathrm{CO} 2$ apneic threshold in newborn infants: possible relevance for periodic breathing and apnea. J Appl Physiol (1985). 2005;98(4):1171-6.

29 Hulzebos CV, van Dommelen P, Verkerk PH, Dijk PH, Van Straaten HL. Evaluation of treatment thresholds for unconjugated hyperbilirubinemia in preterm infants: effects on serum bilirubin and on hearing loss? PLoS One. 2013;8(5):e62858. 